\title{
Das Selbst zwischen Bild und Sprache: Marc Chagalls Autobiographie Mein Leben
}

\begin{abstract}
Der Beitrag untersucht das besondere Verhältnis zwischen Text und Bild im autobiographischen Text des Malers Marc Chagall Mein Leben (Ma vie, 1921-1931). Während Chagalls Bilder autobiographische Elemente enthalten, lehnt sich der Maler sprachlich in seiner literarischen Autobiographie an das Bildliche. Der Schreibprozess veranlasst den Künstler dazu, die Beziehung zwischen dem gewohnten Medium der Malerei und der Versprachlichung der Erinnerung zu reflektieren. Unter der Berücksichtigung der Poetik des Künstlers, dessen Leben vom Sprach- und Kulturwechsel geprägt ist, wird sein Sprachverständnis untersucht. Dabei wird aufgezeigt, dass Bilder bei Chagall als Speicher des Erlebten und die Bildsprache als eine universelle Sprache verstanden werden. An den Besonderheiten des Inhalts und der Form zeigt der Beitrag, dass Mein Leben ein autofiktionaler Text ist, in dem die Auflösung der Grenzen zwischen Realität und Fiktion, Text und Bild konstitutiv ist.
\end{abstract}

Keywords: intermediale Autobiographie; Autofiktion; Bildsprache; Bild und Text; universelle Sprache; Marc Chagall

Bild und Sprache stehen in der autobiographischen Selbstdarstellung in einer produktiven, wechselseitigen Beziehung. Bilder und Photographien werden in den autobiographischen Texten verwendet, während die autobiographische Malerei narrative bzw. textuelle Elemente enthalten kann. Die Autobiographie wird als eine verwandte Form des Sehens und als „das Auge des Ich“ bezeichnet. (Blazejewski 2002, 87-88) Die Geschichte des Selbstporträts zeigt einzelne und serielle Selbstdarstellungen, die als Formen der Selbstbeobachtung, des Tagebuchs bei Dürer, Rembrandt, Vincent van Gogh, Egon Schiele und anderen auftreten und die sich bis in die Gegenwart des photographischen Selbstporträts und der multi- und intermedialen Selbstdarstellung verfolgen lassen.

Der Vergleich mit dem Selbstporträt ist in der Theorie und Praxis literarischer Selbstdarstellung geläufig. Die Autobiographie wird mit dem künstlerischen Selbstporträt verglichen, und trotz der Diskussion um die Möglichkeiten der Analogie und der Komplikationen des Vergleichs der verschiedenen Zeichensysteme (Schmitz-Emans 1999a, 2-16; 1999b, 17-34) werden beide Medien der Selbstdarstellung aufeinander bezogen. Interessant ist in dieser Hinsicht Michel Beaujours Essay Miroirs d'encre (vgl. Beaujour 1980), in dem das literarische Selbstporträt, 
ausgehend von Lessings Medienästhetik in Laokoon, als ein Darstellungsmodus verstanden wird, in dem Inhalte nebeneinander angeordnet sind und der demnach keine retrospektive Erzählung, sondern einen bildähnlichen Versuch darstellt, Momente der Selbstbetrachtung zu erfassen. An Texten wie Michel Montaignes Essais (1572-1592), Rousseaus Rêveries du promeneur solitaire (1776-1778), Nietzsches Ecce Homo (1888-1889), Michel Leiris' L'âge d'homme (1939) und Règle du jeu (1948-1976) postuliert Beaujour das literarische Selbstporträt als eine von der Autobiographie abweichende Gattung. In den 1920ern und 1930ern fangen Autobiographien von Schriftstellern und Künstlern an, mit „Selbstporträt“ überschrieben zu werden. (Hall 2016, 232)

Für Marc Chagalls Gesamtwerk ist das Zusammenspiel der beiden Medien charakteristisch. Er integrierte Sprach- und Schriftelemente in seine Kunst, malte für das Theater und schuf Illustrationen zu literarischen Texten. Neben den zahlreichen Selbstporträts, die seine Biographie, den Schaffensprozess, das Selbst des Künstlers reflektieren, hatte er 1922-1923 seine Autobiographie Mein Leben (1923) entworfen, zunächst in jiddischer Sprache und in einer Version auf Russisch, die die Frau des Künstlers Bella Rosenfeld ins Französische übersetzte. ${ }^{1}$ Der Text umfasst Chagalls Geburt und Kindheit im jüdischen Stadtteil von Witebsk in Weißrussland, sein Studium in St. Petersburg und Paris, die Reise in die Heimat und die Jahre in der Sowjetunion bis zu seiner Rückkehr nach Europa, und greift thematisch Rituale, alltägliche Situationen und Ereignisse auf, die auch in einer Reihe von Chagalls Bildern Ausdruck finden. ${ }^{2}$

Ausgehend von den vorhandenen Untersuchungen zum intermedialen und fiktionalen Charakter der Autobiographie Marc Chagalls werde ich deren intermediale Makro- und Mikrostruktur und Ikonographie aufgreifen, um 1) die Beziehung zwischen Bild und Sprache in Mein Leben zu präzisieren, 2) die Rolle der Sprachlosigkeit und der Sprache zu bestimmen und 3) den Text als ein autofiktionales literarisches Selbstporträt zu deuten.

Der Literaturwissenschaftler Benjamin Harshav hat als einer der Ersten die intermediale Wechselbeziehung zwischen Chagalls bildnerischem und literari-

1 Die russische Version ist verschollen und die französische Version, obwohl von Chagall beaufsichtigt und autorisiert, hält Harshav - genauso wie alle weiteren Übersetzungen - für weniger authentisch als die jiddische Version der Autobiographie, an der Chagall auch nach dem Erscheinen von Mein Leben weiter arbeitete. (Vgl. Harshav 2004, 81) Ich zitiere im Folgenden die deutschsprachige Version (vgl. Chagall 1959), nach einem Abgleich mit der englischsprachigen Übersetzung Harshavs, My Own World (vgl. Harshav 2004, 85-166), und der französischen Übersetzung Bella Chagalls, Ma vie (vgl. Chagall 1957).

2 Näheres zu den Bezügen zwischen Chagalls Leben und dem bildnerischen Werk, zum intertextuellen und kulturellen Hintergrund seiner Autobiographie vgl. Harshav 2004; Schulze 2005; Koller 2012. 
schem Werk wissenschaftlich beschrieben. Er bezieht die ursprünglich poetologische Kategorie des Fiktionalen auf Chagalls Mal- und Erzählweise. In seiner bedeutenden Monographie zu Chagall (2004) betont er neben der erzählenden Malweise gleichzeitig die malerische Erzähltechnik in Mein Leben. Er stellt fest, dass Chagall beim Schreiben dem Diktat seines besonderen malerischen Sehens folgt und den größten Teil des Textes in painterly vignettes, in malerischen Miniaturbildern, präsentiert. Die autobiographischen Fragmente werden von Chagall nicht bloß erzählt, sondern vielmehr visualisiert. (Vgl. Harshav 2004, 70-85)

Der Text von Chagalls Autobiographie ist vielschichtig, was die Bildlichkeit betrifft. Sabine Koller stellt in ihrer Arbeit Marc Chagall. Grenzgänge zwischen Literatur und Malerei (2012) drei Ausdrucksformen der Intermedialität fest: 1) Radierungen als Illustrationen zu konkreten Textstellen, 2) die Inhaltsebene mit der Bezugnahme auf diverse Kunstrichtungen, -werke, -techniken, -epochen der Malerei und der autoreferenziellen Bezugnahme auf eigene Bilder; und 3) die intermediale Stilistik auf der sprachlichen Ebene, das sogenannte pikturale Schreiben. (Vgl. Koller 2012, 49-50) Aspekte des pikturalen Schreibens und die Stellung, die die Sprache in Mein Leben in ihrer visualisierenden und fiktionalisierenden Funktion einnimmt, hängen mit der Spezifik von Chagalls Malerei eng zusammen.

Als Künstler fühlte sich Chagall keiner künstlerischen Richtung zugehörig, obwohl er Züge vieler Bewegungen der europäischen Avantgarde annahm und neu zusammensetzte. Dieser „postmoderne Stil in der Moderne“ wird als demonstrative eclecticism bezeichnet (Harshav 1992), da er sich durch ein Nebeneinander verschiedener Richtungen auszeichnet und trotzdem dank seiner eigenen Bildsprache homogen wirkt. Im Unterschied zum Surrealismus soll diese Bildsprache nicht aus dem Traum bzw. Unterbewusstsein entstanden sein, sondern aus dem tatsächlich Erlebten, während sie durch ihre Opposition zur Mimesis, durch gelöste Realitätszusammenhänge, traumhafte Kompositionen, Autonomie der Formen und Farben keine kunstsprachlichen Neuerungen, sondern eine innovative Fiktion in der Kunst vorstellen. Harshav deutet Chagalls Bilder thematisch und kompositionell als introspektive Darstellungen der sozialen und persönlichen, biographischen Traumwelt. Er positioniert den Künstler Chagall irgendwo zwischen dem Maler des chaotischen Unbewussten und dem naiven Erzähler einer seltsamen Biographie. (Vgl. Harshav 1992; 2010, 70)

Chagalls künstlerische Identität wurde von seinem interkulturellen Lebenslauf geprägt, besonders durch ostjüdische, russische und europäische Einflüsse. Der Maler unterhielt enge Beziehungen zur jiddischen Kultur, und so trägt die Semantik der jiddischen Sprache in zahlreichen Bildern des Künstlers zu deren Lesbarkeit bei. Koller untersucht ausführlich Chagalls Werk, darunter auch seine Selbstporträts, und zeigt, dass der Künstler Sprachbilder in „sprechende Bilder“ 
verwandelt und dass es sich in den meisten Fällen um Implikationen handelt, die man nur mit der Kenntnis der jiddischen Kultur und Sprache entschlüsseln kann. (Koller 2012, 64-104) In seiner Illustration Selbstporträt mit dem Haus (1922) stellt Chagall beispielsweise sich selbst mit dem Haus auf dem Kopf dar, das als Symbol für Heimat steht und die jiddische Redewendung „etwas im Kopf haben“ buchstäblich verbildlicht und gleichzeitig fiktionalisiert, da die Darstellung als absurd oder phantastisch wahrgenommen wird. (Koller 2012, 101) Das Erzählende im Bild Chagalls unterscheidet Koller in Narration (vertreten durch Bildsujet, Bildorganisation und -komposition, die räumliche Gestaltung) und Deskription (in Form von Metapher). (Vgl. Koller 2012, 11)

An der Ikonographie des autobiographischen Gemäldes Ich und das Dorf (1911) lassen sich narrative und fiktionale Züge der künstlerischen Sprache Chagalls verdeutlichen. Die Struktur des Bildes stellt eine Art Kaleidoskop, ein System von verschiedenen geometrischen Formen dar, ein simultanes Nebeneinander von räumlich auseinanderliegenden Dingen und Figuren. Das Gemälde präsentiert verschiedene farbliche Zuordnungen und Narreme ${ }^{3}$ : 1) Ein grünes männliches Profil erstreckt sich über den gesamten rechten Bildrand als Selbstdarstellung des Künstlers, und am linken Rand schaut ein Tierprofil ihm in die Augen. Die Hand des Mannes, die von unten ins Bild kommt, hält eine Phantasiepflanze dem Tier entgegen, auf einem Finger trägt der Mann einen Ring. Die Augen des Tieres und des Mannes sind durch eine feine Linie verbunden. Das kleinere rundliche Licht in den Augen des Tieres scheint dabei ein größeres Leuchten in den Pupillen des Mannes auszulösen, oder möglicherweise spiegelt es sich vergrößert als ein leuchtender Himmelskörper in den Augen des Mannes wieder. 2) Im Profil des Tieres wird eine Melkszene präsentiert, als ein Bild im Bild, mise en abyme, welches das Tiermotiv wiederholt. 3) Im Hintergrund sehen wir eine Häuserreihe und eine dörfliche Szene: ein sich bewegendes Paar, wobei die Figur der Frau und die Häuser, an denen sie vorbeiläuft, kopfüber stehen, eventuell als die Entsprechung des Idioms „auf dem Kopf stehen“. Ein Pope schaut aus der Kirche dem Paar zu.

Die Zusammenhänge des Bildes sind angedeutet und gleichzeitig aufgelöst; die Farben sind nicht realistisch, sie definieren einzelne Bereiche. Die dargebotenen Motive rufen diverse Assoziationen hervor und regen dazu an, ihre Relation und Bedeutung zu deuten. Das Bild ist ein Erinnerungsmosaik, ein Tribut des Künstlers an seine Heimat, wie fast sein gesamtes Werk, es trägt selbstreflexive

3 Ich beziehe mich hier auf Werner Wolf, der den transmedialen Begriff ,Narrem‘ für Erzähleinheiten wie inhaltliche Formen (Zeit, Ort, Figuren, Geschehen, Kausalität) oder Syntaxregeln des Narrativen vorgeschlagen hat. (Vgl. Wolf 2002, 23-103) 
Züge. Das Tier repräsentiert das Dorf und ist die Braut, der Künstler trägt einen Verlobungsring, so dass es sich um eine Vermählungsszene handelt. Die Phantasiepflanze kann als ein Lebensbaum, der Kreis im Zentrum des Gemäldes, der die Mundpartien des Mensch-Tier-Paares verbindet, als der Kreis des Lebens, der Kommunikation, der Kunst verstanden werden. Die Augen des Tieres könnten die Quelle der künstlerischen Inspiration bedeuten, der Künstler reflektiert und vergrößert deren Licht. Die Sprache konstituiert das Bild durch die Metaphorik mit, die Kommunikation zwischen den zentralen Figuren geschieht im Visuellen: durch die Augen, den Blick.

Die narrativen Einheiten, die sich in Chagalls Bildern feststellen lassen, sind mit der Erzählweise seiner Autobiographie verwandt. Ähnlich wie im oben beschriebenen Bild wird der Text nicht entlang der Ereignisse ausgerichtet, sondern stellt eine Anordnung von Stimmungen und Porträts mit einer starken Ausrichtung auf Gefühl, Inspiration und Selbststilisierung als Künstler dar. Die Intermedialität des Textes ist unter anderem transformativ, die Sprache des Künstlers schöpft Farben und Techniken der Darstellung aus seiner Kunst. Chagall schreibt in der Sprache seiner Bilder. ${ }^{4}$ Chagall verwendet das Vokabular eines Malers: Er schreibt nicht nur, sondern er ,skizziert‘, ,vollendet eine Silhouette‘, ,porträtiert‘. (Chagall 1959, 8) Doch so gewohnt wie das Malen gestaltet sich der Schreibprozess nicht. Die Sprache des Künstlers scheint sich den Weg durch das visuelle Denken erkämpfen zu müssen:

Es wäre reizvoller, meine Schwestern und meinen Bruder zu malen.

Wie gern würde ich mich verführen lassen von der Harmonie ihres Haars, ihrer Haut, wie eilig wollte ich in sie hineinspringen, Leinwände und euch selbst berauschen mit der Ausdünstung meiner tausendjährigen Farben!

Doch sie beschreiben? Ich will auch nicht mehr als zwei Worte über meine Tanten sagen.

(Chagall 1959, 18)

Es ist alles, was Chagall an dieser Stelle über seine Geschwister sagen kann, er vermag es nicht, sie mit Worten $\mathrm{zu}$ beschreiben. Im Prozess der Erinnerung wird Chagall mit imaginären Bildern konfrontiert, und er versucht, sie in Worten festzuhalten. Dazu greift er auf Bildevokation, Ekphrasis, Werke anderer Maler und autoreferentielle Verweise auf eigene Bilder zurück, wie beispielsweise in der Beschreibung seines Vaters:

4 Zur Diskussion über die „Sprache“ der Bilder vgl. Schmitz-Emans 1999a, 6-13; und Koller 2012, 59. 
Habt ihr manchmal auf Florentiner Bildern einen von diesen Leuten gesehen, mit nie geschorenem Bart, mit Augen, braun und zugleich wie Asche, mit einem Gesicht von gebranntem Ocker, mit Runzeln und Falten bedeckt?

Das ist mein Vater. (Chagall 1959, 7)

In der Autobiographie folgt Chagall einem besonderen Kompositionsschema. Während im letzten Drittel der Autobiographie das simultane, bildhafte Erzählen nachlässt und das Narrative mit dem Bericht über die politische Situation in Russland immer gewichtiger wird, fügt sich die Sprache bis dahin dem Bildlichen. Unter den evozierten Bildern überwiegen dabei Porträts, alltägliche Rituale und Szenen. Die begleitenden Radierungen betonen die führende Position des Bildlichen im Text. Im ersten Drittel des Textes scheinen die Gestalten der Vergangenheit den Erzähler wie Geister zu bedrängen. Oft bricht er das literarische Porträtieren ab, denn der Nächste wartet darauf, dass sein Porträt versprachlicht wird. Das vertraute Medium der Malerei bietet Chagall die gewohnte Art und Weise, Inhalte einzufangen. Immer wieder betont er den Vorteil des Visuellen vor dem Sprachlichen. Zur Figur des Vaters schreibt er weiter:

Du weißt noch, ich habe von dir eine Studie gemacht. [...]

Darf ich von meinem Vater sprechen?

Was gilt ein Mann, wenn er nichts gilt? Wenn er unschätzbar ist? Und daher fällt es mir schwer, die richtigen Worte für ihn zu finden. [...]

Alles schien mir Rätsel und Traurigkeit an meinem Vater. Unzugängliches Bild. Er war müde, sorgenvoll, nur seine Augen hatten einen milden Glanz von grauem Blau.

(Chagall 1959, 7-8)

Nicht erst die sprachliche Realisierung des Porträts scheitert daran, das innere, durch die Wahrnehmung erzeugte und durch die Erinnerung modifizierte Bild zu realisieren, sondern auch die Kunst, die auch nur eine Annäherung an ein ,unzugängliches Bild“ sein kann. Doch während eine Studie zum Porträt des Vaters als eine selbstverständliche Art der künstlerischen Produktion erscheint, wirft seine Transformation ins Sprachliche die Frage nach Möglichkeit und Legitimation so einer Beschreibung auf. Das gewohnte Übertragen des Denkens in Bilder beeinflusst hier die Sprache, erzwingt das pikturale Schreiben. Der Erzähler tastet sich in seiner Erzählabsicht an den Bildern entlang. Seine Darstellungen von Ereignissen und Personen gehen darauf zurück, so dass die Sprache sich dem Diktat des Visuellen fügt. Der Abschnitt, in dem die Erzählerstimme sich im Dialog an die auftauchenden Geister wendet, wird wie folgt abgeschlossen:

Eure Steine. Eure Gräber, Hecken, trüber Fluß, sanfte Gebete, das alles ist mir vor Augen. Worte gar nicht. Das alles steckt in mir, verborgen, wirbelt und schwebt als Erinnerung. [...] Doch genug! Auf Wiedersehen! (Chagall 1959, 22) 
An dieser Stelle bekennt sich der schreibende Maler wieder dazu, dass er seine Vergangenheit in Bildern und nicht sprachlich abrufen kann.

Auch im weiteren Text, der eine zentrale Position, die Mitte der Autobiographie einnimmt und Chagalls Weg zum Künstler enthält, werden Erinnerungsbilder heraufbeschworen und versprachlicht. Chagall führt sie ein, skizziert sie in Form und Farbe, um sie anschließend um die Emotion, die sie begleitet und kommentiert, zu ergänzen. Das imaginäre Bild ist die Initiation, selbst wenn es zum großen Teil in der Imagination des Erzählers und für den Rezipienten unsichtbar bleibt. So wird die Szene der Geburt des Bruders dargestellt, der Erzähler ist ein ,stillschweigender‘ Beobachter dabei:

\footnotetext{
Mama, halbnackt, liegt im Bett, blaß, zartrosa. Mein jüngster Bruder kam zur Welt.

Die Tische weiß gedeckt.

Rauschen von heiligen Gewändern. [...]

Ich bin traurig. Stillschweigend sitze ich neben den anderen, kaue den Kuchen, die Heringe und das Würzbrot. (Chagall 1959, 45-46)
}

Die Knappheit der Beschreibungen entspricht dem Rhythmus der sich abwechselnden Erinnerungsbilder. Ein anderes Beispiel ist das Fragment, in dem der Tod der Schwester beschrieben wird:

\footnotetext{
Ihre Augen füllten sich mit himmlischem Blau, mit dunklem Silber. Ihre Pupillen erstarrten. Fliegen setzten sich auf ihre Nase. Niemand jagte sie fort. [...]

Das Gefühl: in einigen Stunden wird dieser kleine Körper in der Erde liegen, und die Menschen werden mit ihren Füßen darüber hinstampfen! (Chagall 1959, 60-61)
}

Im Verlauf der ganzen Autobiographie wird die Vergegenwärtigung des Bildes oft durch den spontanen Wechsel ins Präsens realisiert:

\footnotetext{
Ohne ihre Haltung zu verändern, mit geschlossenem Mund und kaum bewegten Lippen, die spitze Frisur genau an ihrem Platz, stellte sie ihre Fragen, schwieg oder sprach sie wie eine Königin. Aber niemand ist da. Nur ich hörte ihr zu, von weitem.

Sie bat mich:

,Mein Sohn, sprich mit mir.‘

Ich bin ein Kind und Mama eine Königin. Wovon denn sprechen? (Chagall 1959, 12)
}

In allen oben zitierten Auszügen fällt die Thematisierung des Unsagbaren und der Stummheit auf. Diese nachdrückliche Betonung der Sprachlosigkeit bei einem Künstler, dessen Leben von drei Sprachen (Jiddisch, Russisch und Französisch) geprägt war, zieht sich durch den ganzen Text und hat eine gewichtige Bedeutung für Chagalls Sprachverständnis. Obwohl jede Sprache samt ihrem kulturellen Kontext für ihn zu einer Heimat, zum Teil seiner Biographie und seiner Identität 
geworden ist, ${ }^{5}$ wird die Sprache der Kunst, die für Chagall die Essenz seines Lebens und seines Selbst spiegelt und eine universelle Sprache bedeutet, zur eigentlichen Heimat des Künstlers. Chagall, der im Laufe seines Lebens mehrmals die weißrussische Heimat verlassen und 1941 auch aus seiner neuen Heimatstadt Paris in die USA ausreisen musste, verwurzelt seine Identität in der Kunst. Der Sprach- und Kulturwechsel ist wichtig für Chagalls künstlerisches Selbstverständnis, er bestimmt seine Suche nach einer konstanten Sprache, die über diesen Wechsel hinaus funktioniert. Die Quelle seiner Poetik der universellen Sprache bleibt im Lebensabschnitt verankert, in dem er seine Selbst-Werdung als Künstler erlebt. Daher wird dieser Lebensphase die Position des ,goldenen Schnitts ‘ im gesamten Text der Autobiographie zugewiesen. Sie erstreckt sich ungefähr vom Ausruf eines Kameraden („Hör mal, du bist ja ein richtiger Künstler!“ [Chagall 1959, 52]), bis zur Teilnahme Chagalls an der Ausstellung in Paris und bis zum Gespräch mit Apollinaire, in dem Chagall seine Position zur Kunst bezieht und so das Selbstbewusstsein als Künstler beweist. (Vgl. Chagall 1959, 108-113)

Chagall distanziert sich in Paris von den modernen Kunstrichtungen und deren Regeln, die ihn „traurig und verkrampft“ machen (Chagall 1959, 109). Er begreift die Kunst als einen Seelenzustand (vgl. Chagall 195, 113), als eine „blaue“, über seine Bilder, auf seine Leinwand fließende Seele (Chagall 1959, 108), und die „Seele eines jeden ist heilig, eines jeden Zweibeiners an jedem Punkt der Erde“ (Chagall 1959, 113). ${ }^{6}$ Die ,Sprache der Kunst“ wird damit als ein universales Medium begriffen, und die Besonderheit der künstlerischen Sprache Chagalls ist der Versuch, die „eigene Logik“, die „eigene Vernunft“ und die Freiheit des Ausdrucks zu erreichen. (Chagall 1959, 113) Ich möchte Harshavs Begriff demonstrative eclecticism aufgreifen, den ich oben erwähnt habe. Entlehnungen aus verschiedenen Kunstrichtungen und die eigene Bildsprache, die auf Erinnerung und die drei kulturellen und sprachlichen Räume seiner Biographie zurückzuführen sind, gelten auch für die Sprache seiner Autobiographie.

Chagall verwischt im Text die Grenzen zwischen Medien und Stilrichtungen. Auch die Grenzen zwischen der Realität und der Traumwelt, wie bereits Harshav hervorhebt, werden bei Chagall aufgehoben. Von den ersten Sätzen der Autobiographie an bedingt die Anlehnung des sprachlichen Ausdrucks an das Bild die autofiktionale Form: „Was mir zuerst in die Augen sprang, war ein Trog. Einfach,

\footnotetext{
5 Im autobiographischen Gemälde Kubistische Landschaft (1918), das Chagalls Weg durch sprachliche und kulturelle Stationen reflektiert, verwendet er als Textelement das sprachliche Spiel mit seinem Nachnamen, der im Russischen „schritt“ bzw. „ging“ bedeutet und den er nach der Zahl der Jahre wiederholt, die er in einer bestimmten Sprache verbracht hatte. (Vgl. Harshav 2004, 15) 6 „,U]ne âme bleue, jailissant sur mes toiles“ (Chagall 1957, 155); „L’âme de tous est sainte, de tous les bipèdes sur tous les points de la terre“. (Chagall 1957, 160)
} 
wuchtig, halb hohl, halb oval. Ein Trog vom Trödelmarkt. Einmal drin, fühlte ich ihn ganz aus.“ (Chagall 1959, 5)

Chagall kann sich natürlich nicht an den Trog erinnern, in den man ihn als beinahe totgeborenes Baby hineintaucht, um ihn $\mathrm{zu}$ beleben, doch vom ersten Satz an lädt er den Leser dazu ein, mit ihm ,hinzusehen', und skizziert das Erscheinungsbild des Trogs. Der Schreibakt wird zum performativen Akt des Malens, die Lektüre zum Akt des performativen Sehens. Das dargebotene Bild hat keinen Anspruch auf die Faktualität, die Tatsachen im Text haben keinen dokumentarischen Charakter. Von Anfang an wird ein Bild geboten, das um das Erfundene bewusst erweitert ist. Das Sehen bezieht sich auf das innere, imaginäre Bild, das der Künstler sprachlich inszeniert, dadurch werden die Imagination und der Blick als ein performativer Akt in den Prozess der Lektüre miteinbezogen.

Die Frage danach, inwiefern die erfundene autobiographische Wirklichkeit den tatsächlichen Erfahrungen entspricht, und das konstitutive Moment der Autobiographie in ihrem Kunstcharakter sind für die Bestimmung des Textes als Autofiktion entscheidend. (Vgl. Wagner-Egelhaaf 2005, 41) Der Kunstcharakter der Gattung Autobiographie ist viel diskutiert worden. Roy Pascal steht am Anfang dieser Diskussion, die die Durchlässigkeit der Grenzen zwischen Literarizität und Fiktionalität der Autobiographie herausstellt. (Vgl. Pascal 1960) Autofiktionale Verfahren sind nicht nur für literarische Texte, sondern für alle Selbstdarstellungsformen relevant, auch für die Malerei und die Photographie. Laut der Unterscheidung der autofiktionalen Formen (Zipfel 2009, 284-314), handelt sich hier um eine Autobiographie, in der sich das Fiktionale durch den expliziten Konstruktionscharakter bemerkbar macht. Im Gegensatz zum chronologischen bzw. dialektischen Fortschreiten des narrativen Erzählens findet der Text in der Zeit des Schreibens statt: Das erinnernde Subjekt tritt aus der linearen Zeit heraus, die fragmentarische Erzählweise des Textes spiegelt die assoziativ rekonstruierte, auf Bildern aufgebaute Erinnerung. Makrostrukturell gesehen folgt der Text zwar der Chronologie des dargestellten Lebensabschnittes, in seiner Mikrostruktur löst er sich jedoch von den realistischen Zusammenhängen ab. Dabei werden die Grenzen zwischen dem Alläglichen und der Imagination, zwischen Leben und Kunst aufgehoben:

Hinter dem Fenster - die Nacht.

Nur der Pope schläft und hinter ihm, hinter seinem Haus, die Leere, die Geister.

Aber mein Onkel spielt Geige. [...]

Nur mein Kopf schwebt leise durchs Zimmer.

Die Decke wird durchsichtig. Wolken und blaue Sterne dringen herein, zugleich mit dem Geruch von Äckern, Stall und Straßen.

Ich will schlafen. [...]

Ein See. Seine Töchter grasen wie rote Kühe. (Chagall 1959, 20-21) 
In der Sprache verschwimmen hier Erinnerung, Traum und Imagination: Die Geister, der schwebende Kopf, die roten Kühe und die gelösten Zusammenhänge sind als Motive aus Chagalls Bildern erkennbar, und die komprimierte Ausdrucksweise lässt Leerstellen frei, die die Differenz zwischen dem Sichtbaren/Genannten und dem Unsichtbaren/Ungesagten bestimmen.

Die Poetik Chagalls bezieht die Idee des Lebens in der Kunst und des Künstlers als Kunstwerk und der vollkommenen Hingabe mit ein. In der imaginären Ansprache an seinen ersten Kunstlehrer kommt dieser Gedanke zum Ausdruck: „Ich brauche keinen Ruhm [...]; wie Ihre Bilder aufgehängt sind, will ich selbst hängen in Ihrer Straße, in Ihrer Nähe [...]. “ (Chagall 1959, 59) Mein Leben enthält einige sprachlich entworfene Selbstporträts, unter anderem zwei photographische, die man nicht übersehen darf. Sie tragen zur Inszenierung des Selbst als Kunstwerk bei. Das erste Selbstporträt entsteht beim Besuch eines Photographen, bei dem die ganze Familie anwesend ist, und zeigt ein beinahe filmisches Standbild. Diese Strategie des Anhaltens des Erzählflusses, um ein Bild einzuführen, erinnert an ein Standbild und wird von Chagall viel verwendet: „Ich war ein Kind von fünf, sechs Jahren, bekleidet mit einem Kittel von rotem Samt mit goldenen Knöpfen, und hielt mich dicht an Mamas Rock. Genau wie meine Schwester, die auf der anderen Seite stand, hielt ich den Mund offen, um besser atmen zu können.“ (Chagall 1959, 64)

Das andere Selbstporträt wird zunächst als Narziss, als ein Jünglingsgesicht vor dem Spiegel stilisiert (vgl. Chagall 1959, 68). Danach geht Chagall von der Selbstbetrachtung zur Idee der Selbstdarstellung als ein gemaltes Porträt über. Schließlich überlagert er das Selbstporträt ironisch mit der Photographie, indem er auf die Technik des verschönernden Retuschierens anspielt, die er während seiner Lehre beim Photographen an den photographischen Porträts seiner Bekannten widerwillig ausführte (vgl. Chagall 1959, 63): „Ich gebe zu, ich zögerte nicht, mir etwas Schatten um die Augen zu legen, den Mund leicht zu röten, obwohl ich es gar nicht nötig hatte [...]. “(Chagall 1959, 69)

Das physische Erscheinungsbild verwandelt sich an dieser Stelle aus dem Objekt der Betrachtung in ein Kunstobjekt, gleichzeitig wird es zu einem sprachlichen Bild. Diese Überlagerung der Medien und die Überlagerung des Lebens mit der Kunst kommt im Abschlussabschnitt, in den letzten Sätzen der Autobiographie, programmatisch zur Sprache:

Diese Seiten haben dieselbe Bedeutung wie eine bemalte Leinwand.

Wenn es in meinen Bildern ein Versteck gäbe, könnte ich sie dort hineinschieben... Oder soll ich sie lieber irgendeiner Figur von mir auf den Rücken kleben oder gar dem ,Musikanten` meiner Wandmalerei auf die Hosen?... (Chagall 1959, 173) 
Das Wesen des Autobiographischen in Mein Leben ist demnach mehr als ein intermediales Werk, in dem graphische, mentale und verbale Bilder miteinander kombiniert werden. Das Sprachliche und das Bildliche gehen hier im produktiven Miteinander auf, denn die Sprache nährt sich von den Bildern, die den Anlass geben zu erzählen und sich als universelle Speicher des Erlebten, des autobiographischen Selbst erweisen. Die Sprache führt uns den Wechsel bzw. die Überlagerung der Medien vor. Obwohl die narrativen textuellen Formen in der Poetik Chagalls dem Visuellen gegenüber zweitrangig und betont unzulänglich sind, entwickelt der Künstler im Prozess der Versprachlichung des Bildes bzw. der Verbildlichung der Sprache ein literarisches Selbstporträt, eine Autofiktion, in der Text und Bild, Realität und Fiktion fließend ineinander übergehen.

\section{Literaturverzeichnis}

Beaujour, Michel. Miroirs d'encre: Rhétorique de l'autoportrait. Paris: Éditions du Seuil, 1980.

Blazejewski, Susanne. Bild und Text - Photographie in autobiographischer Literatur. Würzburg: Königshausen \& Neumann, 2002.

Chagall, Marc. Ma vie. Dijon: Stock, 1957.

Chagall, Marc. Mein Leben. Stuttgart: Hatje, 1959.

Harshav, Benjamin. Marc Chagall and the Jewish Theater. New York, 1992 https://archive.org/ stream/chagallj00chag/chagallj00chag_djvu.txt (14. Juli 2016).

Harshav, Benjamin. Marc Chagall and His Times. A documentary narrative. Stanford: Stanford University Press, 2004.

Hall, James. Das gemalte Ich. Die Geschichte des Selbstporträts. Darmstadt: Philipp von Zabern, 2016.

Koller, Sabine. Marc Chagall. Grenzgänge zwischen Literatur und Malerei. Köln, Weimar, Wien: Böhlau, 2012.

Pascal, Roy: Design and Truth in Autobiography. London: Routledge and Kegan Paul, 1960.

Schmitz-Emans, Monika. Die Literatur, die Bilder und das Unsichtbare. Spielformen literarischer Bildinterpretation vom 18. bis zum 20. Jahrhundert. Würzburg: Königshausen \& Neumann, 1999a.

Schmitz-Emans, Monika. „Das visuelle Gedächtnis der Literatur. Allgemeine Überlegungen zur Beziehung zwischen Texten und Bildern." Das visuelle Gedächtnis der Literatur. Hg. Manfred Schmeling und Monika Schmitz-Emans. Würzburg: Königshausen \& Neumann, 1999b. 17-34.

Schulze, Theodor. „Abendmahl und Schlachtopfer. Übergänge zwischen Leben, Ritual und Ikone in Autobiographie und Bildern von Marc Chagall." Ikonologie des Performativen. Hg. Christoph Wulf und Jörg Zirfas. München: Wilhelm Fink, 2005. 104-130.

Wagner-Egelhaaf, Martina. Autobiographie. Stuttgart, Weimar: Metzler, 2005.

Wolf, Werner: „Das Problem der Narrativität in Literatur, bildender Kunst und Musik: Ein Beitrag zu einer intermedialen Erzähltheorie“. Erzähltheorie transgenerisch, intermedial, interdisziplinär. Hg. Ansgar Nünning und Vera Nünning. Trier: Wissenschaftlicher Verlag, 2002. 23-103. 
Zipfel, Frank: „Autofiktion. Zwischen den Grenzen von Faktualität, Fiktionalität und Literarität?“ Grenzen der Literatur. Zu Begriff und Phänomen des Literarischen. Hg. Simone Winko, Fotis Jannidis und Gerhard Lauer. Berlin und New York: De Gruyter, 2009. 284-314.

Irina Rosenau, M.A., Studium der Germanistik an der Staatlichen Linguistischen Universität in Minsk (1995-2000). Studium der italienischen Sprach- und Literaturwissenschaft, Germanistik und Komparatistik an der Universität des Saarlandes und an der Scuola Normale Superiore di Pisa (M.A. 2008). Lehrbeauftragte für allgemeine und vergleichende Literaturwissenschaft in Saarbrücken. 